\title{
APPORT DE LA MICROSCOPIE ÉLECTRONIQUE SOUS INCIDENCE RASANTE A L'ÉTUDE DES SURFACES, APPLICATION AU LAITON 70/30
}

\author{
E. Darque-Ceretti, M.Y. Perrin et F. Delamare \\ Ecole des Mines de Paris, C.E.M.E.F.', Sophia-Antipolis, \\ 06565 Valbonne Cedex, France
}

Résumé - On montre combien la diffraction des êlectrons (100 keV) sous incidence rasante aìde à la comprêhension des données obtenues par spectrométrie Auger. On prend pour exemple 1 'étude d'une surface oxydée de laiton $70 / 30$. Les résultats sont discutés, et confrontés à ceux obtenus en ESCA. L'influence du frottement sur la composition superficielle est observêe.

Abstract - We point out the complementarity of RHEED and Auger electron spectrometry. An oxidized $70 / 30$ brass surface is taken as example. The results are discussed and compared with ESCA data. Friction is noted to have an influence on surface composition.

\section{I - INTRODUCTION}

L'expêrience montre qu'il est dēlicat de caractériser à l'êchelle du nanomètre les phases tridimensionnelles présentes sur une surface. Or, ces difficultês pourraient bien souvent être surmontées si l'on pouvait obtenir des données structurales.

Le but de ce travail est de rappeler qu'en dehors de la diffraction des électrons lents (LEED), i] existe une méthode commode, la diffraction par réflexion des êlectrons de haute énergie (RHEED) sous incidence rasante /1/ qui peut être utilisée avec succès pour 7 'étude des couches minces (par ex. 12,3/). Cette technique présente les avantages suivants :

- elle fournit des données structurales

- la profondeur du matériau concerné (1 à $5 \mathrm{~nm}$ ) est telle qu'elle se place

à la charnière des matériaux bi- et tri- dimensionnels $/ 2 /$.

- elle s'accommode de substrats polycristallins

- elle utilise un matériel relativement peu sophistiqué et donc peu coûteux.

Cette technique de microanalyse devrait donc rendre de grands services aux laboratoires qui pratiquent l'analyse élémentaire des surfaces, par exemple par spectrométrie des électrons Auger (A.E.S.). Au lieu d'être forcés de tirer des informations complémentaires des superpositions de cartes de répartition d'ëlēments, ou de la simultanéité des variations de profils de "concentrations" élémentaires, les expérimentateurs disposeraient alors de données structurales sur les phases superficielles.

C'est ce que nous nous proposons de montrer en prenant pour exemple l'étude de la surface d'un échantillon de laiton $\alpha, 70 / 30$.

\section{II - LA TECHNIQUE D'EXAMEN}

On utilise un microscope ëlectronique conventionnel (ici, un Philips El:1300) avec un porte-échantillon adapté au maintien d'ëchantillons massifs permettant la diffraction sous incidence rasante $\left(\sim 1^{\circ}\right)$. Seules les lentilles $\mathrm{Cl}$ et $\mathrm{C} 2$ du système d'illumination sont utilisées. Le diamètre du diaphragme objectif (20 um) permet d'atteindre une résolution angulaire d'environ $10^{-5}$ radian. La tension d'accélération est comprise entre 100 et $20 \mathrm{keV}$ suivant que l'on veut voir plus d'anneaux de diffraction ou que l'on désire une meilleure résolution pour les premjères familles de

\footnotetext{
ERA. CNRS $\mathbf{n}^{\circ} 837$
} 
plans diffractants.

Compte tenu de l'angle d'incidence utilisé et du libre parcours moyen des électrons calcuTé par la formule de BETHE /4/, l'épaisseur de la couche participant aux phénomènes de diffraction est de l'ordre de $5 \mathrm{~nm}$ à $100 \mathrm{keV}$ et de $1 \mathrm{~nm}$ à $20 \mathrm{keV}$.

L'aire examinée est une ellipse d'axes $280 \times 30 \mu \mathrm{m}$.

La détermination de la constante de chambre du microscope est obtenue à 1 'aide du cuivre pur. Des étalons de $\mathrm{ZnO}$, CuO et $\mathrm{Cu}_{2} \mathrm{O}$ sont également examinés.

\section{III - ETUDE DE LA SURFACE DE LAITON}

Les mécanismes de l'oxydation des alliages sont parfois complexes, et peuvent rendre la prévision des oxydes réellement formés hasardeuse. L'oxydation du laiton $\alpha, 70 / 30$ à la température ambiante et à l'air a été récemment étudiée par ESCA par divers auteurs, soit sur des échantillons massifs, soit sur des films minces déposés sur acier. Les conclusions peuvent se regrouper selon deux écoles :

- ou bien formation du seul oxyde de zinc Zn0 /5,6/

- ou bien formation simultanêe de Zn0 et des oxydes de cuivre CuO et $\mathrm{Cu}_{2} \mathrm{O} / 7$ à $10 /$

mince) examiné.

Ces conclusions sont indépendantes du type d'échantilion (massif ou film

La surface que nous avons êtudiée est celle d'un revêtement de laiton $\alpha, 70 / 30$ déposé sur acier par électro-codéposition. L'échantillon, plan, a été poli, puís conservé une semaine à l'aìr à la température ambiante en prësence de déshydratant.

Sa surface a été étudiée par spectrométrie Auger et par RHEED ; les technjques complémentaires (ESCA, microanalyseur ionique) ont étē utilisées pour confirmer les résultats obtenus.

La figure 1 montre un spectre Auger dérivé typique obtenu sur la surface. On constate la présence de $\mathrm{Cu}, \mathrm{Zn}$ et 0 , accompagnés de diverses impuretés. L'évolution des "concentrations" en fonction du temps d'érosion ionique (ions $\mathrm{Ar}^{+}$accélérés sous $3 \mathrm{keV}, 70 \mathrm{\mu A} / \mathrm{cm}^{2}$ ) est représentêe dans la figure 2 . Ces "concentrations" sont calculées à partir des hauteurs pic à pic corrigées à la fois de l'effet de numéro atomique $/ 11$ / et de l'effet de pulvérisation prëférentielle (travail en cours). On y constate la similitude des évolutions des profils $Z n$ et 0 . L'interprétation par la présence de $\mathrm{ZnO}$ est tentante, mais peut se révéler beaucoup trop simpliste.
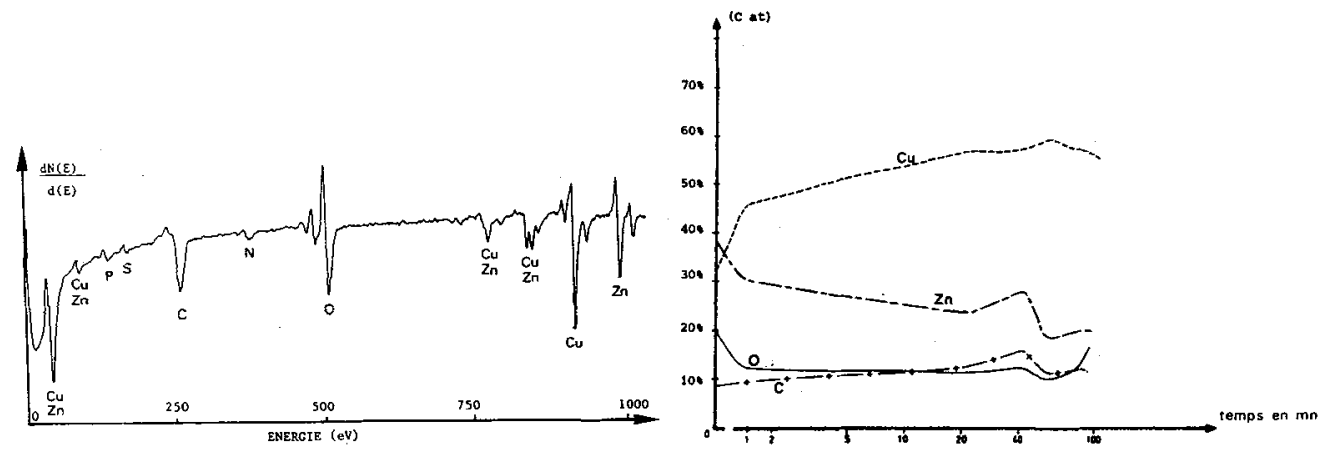

Fig.1- A.E.S. Spectre typique obtenu sur une surface de laiton 70/30 oxydée
Fig.2. - A.E.S. Profils de "concentrations" obtenus sur la même surface. 
L'ESCA a été utilisée pour recouper nos résultats. Cette technique met en évidence la prēsence de $\mathrm{ZnO}$ et d'un peu de $\mathrm{Zn}$ en surface (fig. 3 ). Elle ne détecte ni CuO (absence de satellite sur le pic Cu $2 \mathrm{p}_{3} / 2$, figure 4 ), ni Cu2O (absence de décalage en énergie sur le pic Auger de cuivre, fig. 5).

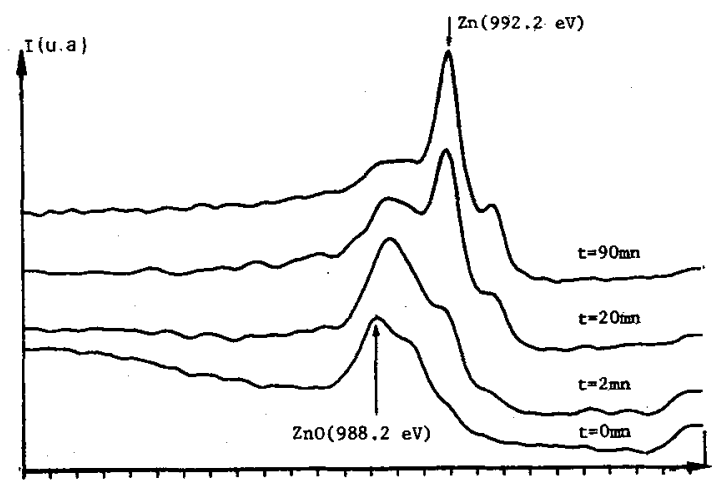

ENERGIE DE LIAISON

Fig. 3 - ESCA - Evolution des pics Auger du $\mathrm{Zn}$ en fonction du temps d'érosion. La proportion $\mathrm{ZnO} / \mathrm{Zn}$ décroît progressivement.

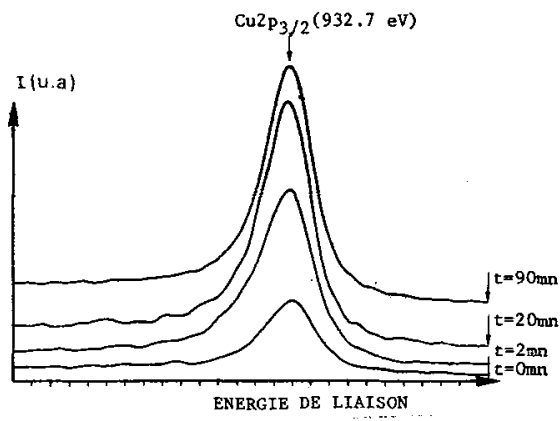

Fig. 4 - ESCA - Evolution du pic Cu2p3/2 en fonction du temps d'érosion. L'absence de satellite prouve la non existence de cuO.

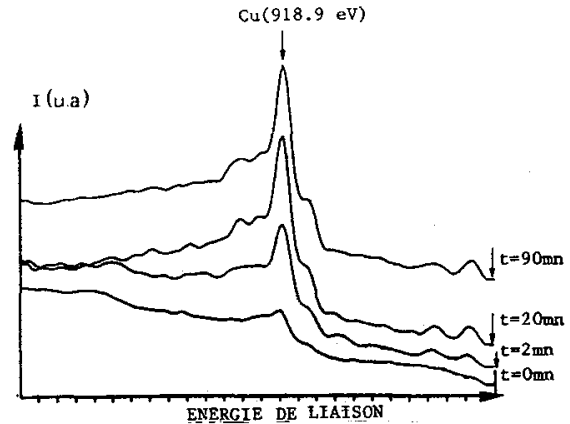

Fig. 5. ESCA - Evolution des pics Auger de Cu en fonction du temps d'érosion. $L$ 'absence de décalage en énergie prouve l'absence de Cu20. 
La figure 6 montre un clichë typique obtenu par RHEED. Les anneaux correspondent soit ầ $\mathrm{ZnO}$ soit au laiton $\alpha$. On ne détecte ni cuivre ni oxyde de cuivre.

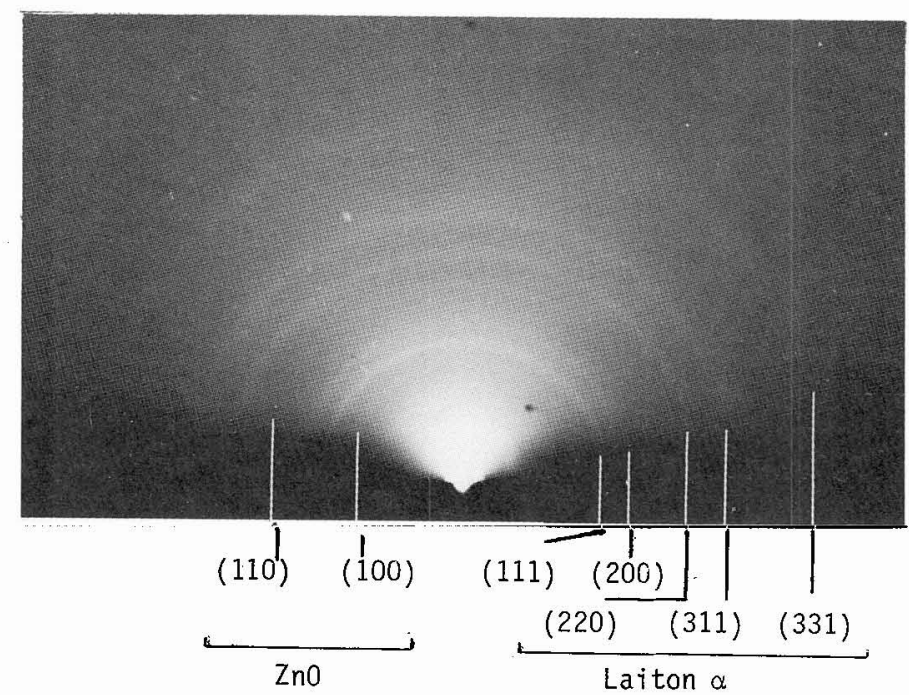

Fig. 6 - RHEED - Clichē de diffraction obtenu à $100 \mathrm{keV}$ sur la même surface initiale.

\section{IV - DISCUSSION}

Etudiée en RHEED (aire d'analyse $280 \times 30 \mu \mathrm{m}$ ) ou par AES (aire analysée : ф5 $50 \mathrm{\mu m}$ ) la répartition de $\mathrm{Zno}$ sur la surface parait homogène. L'ESCA (aire analysêe $\backsim 5 \mathrm{~mm}^{2}$ ) montre la présence d'un très faible signal Zn qui prouve que le taux de recouvrement de la surface en $\mathrm{ZnO}$ est légèrement inférieur à 1 .

Des images en $\mathrm{Zn}^{+}$et $0^{+}$effectuées par spectroscopie de masse au microanalyseur ionique (aire analysêe : $\varnothing 250 \mu \mathrm{m}$; résolution spatiale : $1 \mu \mathrm{m}$ ). montrent qu'après une érosion d'environ $5 \mathrm{~nm}$, i] reste encore un peu de Żno réparti en un petit nombre d'ilots ne couvrant au total que $3 \%$ de la surface.

De la quasi homogénéité du film de $\mathrm{ZnO}$, on peut déduire que le laiton $\alpha$ détecté par RHEED est celui qui est sous 1'oxyde. Compte-tenu de 1 'épaisseur du matériau concerné par les phénomènes de diffraction, et des intensités respectives des anneaux de diffraction, le film de Zno doit avoir une épaisseur de l'ordre de $2 \mathrm{~nm}$. Deux conséquences en découlent :

- on peut donc représenter la répartition des phases superficielles sur le laiton de la manière suivante : Zno taux de recouvrement $\approx 0,95$ (dont $\approx 0,92$ d'épaisseur $\approx 2 \mathrm{~nm}$ et 0,03 d'épaisseur supérieure à $5 \mathrm{~nm}$ ); laiton $\alpha$, taux de recouvrement $\sim 0,05$.

- les profils AES montrent que la vitesse d'érosion de Zno est une fois et demie celle du laiton $70 / 30$ dans nos conditions d'érosion.

En ce qui concerne le laiton a la distance entre plans (111) que nous mesurons est de $0,214+0,001 \mathrm{~nm}$. Comme elle varie linéairement avec la teneur en zinc (10i de Vegard), cē lle-ci peut donc être déterminêe à partir des seules données structurales. On trouve $30 \pm 3,5 \%$ en poids, c'est-à-dire $29 \pm 3,4 \%$ atomique. On ne détecte donc pas de surconcentration en cuivre à l'interface $\mathrm{ZnO} / \mathrm{laiton}$, ce qui est confirmé par les profils de la figure 2. 


\section{$V$ - INFLUENCE DU FROTTEMENT}

Pour observer l'effet de frottement sur la composition superficielle, on étire $T^{\prime}$ échantilion entre des outils, selon une technique décrite ajileurs /12/. Le niveau dess contraintes est imposé par l'obtention d'une faible déformation plastique $\left(\varepsilon \sim 10^{-2}\right)$. Le frottement est celui de 1 'outil sur la surface. Les conditions mécaniques de ce type de contact sont relativement bien connues $/ 12 /$.

Huit passes identiques sont réalisées sur le même échantillion, au même endroit, mais sur des aires décroissant chaque fois. On peut ainsi examiner sur le même échantillon 7 'effet de chaque incrëment de frottement.

Au fur et à mesure que le nombre d'étirages croît, le coefficient de frottement de Coulomb décrồt (de 0,23 à 0,17 ). Simultanēment, on voit disparaître en RHEED les anneaux de diffraction correspondant à ZnO tandis que se renforcent ceux du lajton $\alpha$.

La détection de Zno, qui se faisait sur l'ensemble de la surface de l'échantillon ne se fait plus que sur des plages discrètes, dont le nombre décrôit lorsque croît le nombre d'étirages. L'oxyde Zno disparaît, on ne voit plus alors que les anneaux du laiton $\alpha$.

Rappelons que /13/ avajt noté la transformation sous l'influence du frottement de la phase $\alpha$ en phase $\beta$, dans le biphasé $\alpha+\beta$, tandis que $/ 6 /$ observait $1 a$ transformation inverse.

VI - CONCLUSIONS

D'emploi relativement aisée, la méthode RHEED fournit des données structurales qui se révèlent extrêment utiles pour l'étude des films très minces.

Dans l'étude des surfaces d'alliages constituant des solutions solides, elle peut même, par application de 1a $10 i$ de Vegard, aider à l'étalonnage des techniques spectroscopiques d'analyse de surface.

\section{REMERCIEMENTS}

Nous remercions pour de fructueuses discussions et l'usage de leurs techniques respectives:

- MM. BLAISE (Physique du Solide, ORSAY) et PIVIN (Métallurgie Physique, ORSAY) pour ie microanalyseur ionique.

- M. TRAN MINH DUC (Institut de Physique Nucléaire, LYON) pour l'ESCA.

\section{BIBLIOGRAPHIE.}

/1/ PLUCHERY M., PELISSIER JH., $5^{\text {th }}$ Intern. Congress for Electron Microscopy Academic Press (1962) - JJ4

12/ WERLEN-RUZE B., Thèse Paris (1970)

13/ LAERMANS C., MICHIELS L. and DE BOCK A., Thin solid films, 15 (1973) 317

14/ BETHE K., Ann. Phys. 5 (1930) 325

15/ STORP S., HOLM R., Próc. 7 th Intern. Vac. Congr. and 3rd Intern. Conf. Solid surfaces (Vienna 1977) 2255.

16/ HAEMERS G., MOLLET J., J. of Elastomers and Plastics 10 (1978) 241

17/ VAN OOIJ., Wire Journal 11, 8 (1978) 40

18/ BARR T.L., HACKERBERG. J., Appi. SCi., 10 (1982) 523

19/ MAROIE S., VERBIST J.J., HAEMERS G., les couches minces-survimet 78 (1978) 107

/10/ MAROIE S., Thèse d'Etat (1982) Namur. Facultês Universitaires Notre Dame de la Paix.

111/ PALMBERG P.N. and a1. "Handbook of Auger Electron Spectroscopy". Physical Electronics Ind. Ed. 2è ed. (1976)

112/ DELAMARE F., AYACHE G., et QuANT In J.C., Mém. Et. Sci. Rev. Met. 6 (1983) 303

/13/ JAHANMIR S., Wear, 58 (1980) 387. 